\title{
Design and validation of an oligonucleotide microarray for the detection of genomic rearrangements associated with common hereditary cancer syndromes
}

Debora Mancini-DiNardo, Thaddeus Judkins, Nick Woolstenhulme, Collin Burton, Jeremy Schoenberger, Matthew Ryder, Adam Murray, Natalia Gutin, Aaron Theisen, Jayson Holladay, Jonathan Craft, Christopher Arnell, Kelsey Moyes and Benjamin Roa*

\begin{abstract}
Background: Conventional Sanger sequencing reliably detects the majority of genetic mutations associated with hereditary cancers, such as single-base changes and small insertions or deletions. However, detection of genomic rearrangements, such as large deletions and duplications, requires special technologies. Microarray analysis has been successfully used to detect large rearrangements (LRs) in genetic disorders.

Methods: We designed and validated a high-density oligonucleotide microarray for the detection of gene-level genomic rearrangements associated with hereditary breast and ovarian cancer (HBOC), Lynch, and polyposis syndromes. The microarray consisted of probes corresponding to the exons and flanking introns of BRCA1 and BRCA2 $(\approx 1,700)$ and Lynch syndrome/polyposis genes MLH1, MSH2, MSH6, APC, MUTYH, and EPCAM $(\approx 2,200)$. We validated the microarray with 990 samples previously tested for LR status in BRCA1, BRCA2, MLH1, MSH2, MSH6, APC, MUTYH, or EPCAM. Microarray results were $100 \%$ concordant with previous results in the validation studies. Subsequently, clinical microarray analysis was performed on samples from patients with a high likelihood of HBOC mutations $(13,124)$, Lynch syndrome mutations $(18,498)$, and polyposis syndrome mutations $(2,739)$ to determine the proportion of LRs.

Results: Our results demonstrate that LRs constitute a substantial proportion of genetic mutations found in patients referred for hereditary cancer genetic testing.

Conclusion: The use of microarray comparative genomic hybridization (CGH) for the detection of LRs is well-suited as an adjunct technology for both single syndrome (by Sanger sequencing analysis) and extended gene panel testing by next generation sequencing analysis. Genetic testing strategies using microarray analysis will help identify additional patients carrying LRs, who are predisposed to various hereditary cancers.
\end{abstract}

Keywords: Microarray analysis, Large genomic rearrangements, Hereditary breast and ovarian cancer, Lynch syndrome, Genetic screening

* Correspondence: broa@myriad.com

Myriad Genetic Laboratories, Inc., Salt Lake City, UT 84108, USA 


\section{Introduction}

Sequencing and large rearrangement (LR) analyses detect DNA changes within hereditary cancer genes and are offered to individuals with a personal and/or family history of cancer to identify pathogenic mutation carriers. For example, patients with pathogenic mutations in $B R C A 1$ or $B R C A 2$ have a diagnosis of hereditary breast and ovarian cancer syndrome (HBOC), a condition for which there are extensive medical management guidelines aimed at the prevention and early detection of breast and ovarian cancer. Lynch syndrome is the most common inherited colorectal cancer (CRC) syndrome and is associated with mutations in DNA mismatch repair (MMR) genes, mainly MLH1 and MSH2, but also MSH6, PMS2, and EPCAM [1]. In addition, there is an increased risk for colorectal cancer associated with mutations in the $A P C$ (familial adenomatous polyposis [FAP] or attenuated-FAP) and MUTYH (MUTYH-associated polyposis) genes. Screening is important for genetic counseling in affected families and for early diagnosis or disease prevention in carriers. Early identification of mutation carriers allows for increased clinical surveillance and early detection, and may prompt more aggressive prevention strategies, such as prophylactic surgery or chemoprevention, to reduce risk.

Conventional Sanger sequencing detects the majority of germline mutations associated with hereditary cancers, which consist primarily of nonsense, missense, small in/del and splice-site mutations. However, conventional Sanger sequencing cannot detect certain classes of genetic alterations, including single- and multi-exonic genomic rearrangements such as deletions and duplications. Such genomic rearrangements account for a clinically significant proportion of the common hereditary cancer syndromes. For instance, genomic rearrangements, primarily deletions, account for $5-30 \%$ and $10-60 \%$ of all $M L H 1$ and $M S H 2$ pathogenic germline alterations, respectively, in Lynch syndrome families; the wide range of frequencies is a result of small sample populations in most studies [2-9]. Baudhuin et al. utilized both Southern blotting and MLPA techniques in a consecutive series of 365 unrelated cases and found that, although the majority of pathogenic mutations identified in $M L H 1$ and $M S H 2$ were point mutations and small insertions/deletions, large genomic alterations were present in $17.9 \%$ and $45.3 \%$ of the $M L H 1$ and $M S H 2$ mutation-positive carriers, respectively [10]. Large genomic rearrangements in $B R C A 1$ and $B R C A 2$ account for a low but persistent percentage of all BRCA1 and BRCA2 alterations [11,12]. Genomic rearrangements of $B R C A 1$ appear to be more prevalent than $B R C A 2$ genomic rearrangements [13].

A variety of molecular genetic techniques can be used to detect genomic rearrangements in individuals suspected of a hereditary cancer syndrome, including multiplex quantitative PCR, MLPA, and Southern blotting.
The sequencing of the human genome and the development of high-throughput methods of robotically arraying genetic material on a solid substrate have led to the development of genomic microarrays [14,15], which represent the integration of traditional and molecular cytogenetic techniques and enable the clinical diagnosis of chromosomal abnormalities at an unprecedented resolution. Microarrays comprise thousands of discrete segments of DNA, or probes, selected from genomic regions of interest, from intervals throughout the genome. Microarray analysis has been successfully used to detect changes in DNA copy number in genetic disorders associated with congenital anomalies and in solid tumors [16-18]. Several studies have used microarrays for the identification of genomic rearrangements associated with common hereditary cancer syndromes in a research setting $[19,20]$. Staaf et al. designed a custom oligonucleotide microarray for the characterization of genomic rearrangements of BRCA1, BRCA2, MLH1, and MSH2. The microarray results were concordant with MLPA in 26/30 cases; four discrepancies were the result of inaccurate MLPA results.

In this study, we describe the design and validation of an oligonucleotide-based microarray targeted to genes associated with hereditary cancer. Microarray analysis of patients referred to our laboratory for LR testing in conjunction with sequencing analysis demonstrates that LRs represent a substantial proportion of genetic mutations identified in the clinical laboratory. It should be noted that an increasing number of laboratories have integrated the use of next generation sequencing (NGS) technology for the detection of mutations in panels of genes. Though NGS is a proven technology for the accurate detection of sequencing mutations, detection of LRs by NGS remains challenging [21]. Until we achieve accurate and reliable LR detection by NGS, microarray CGH will remain a viable transitional tool for the detection of LRs for both single syndrome and extended panel testing.

\section{Materials and methods Microarray design}

Our laboratory developed a high-density oligonucleotide microarray consisting of approximately 1,700 overlapping probes corresponding to exons and flanking introns of BRCA1 and BRCA2. Approximately 2200 overlapping probes correspond to exons and flanking introns of MLH1, MSH2, MSH6, APC, and MUTYH, and exons 2, 3, 8, 9, and the 3' UTR of EPCAM. On average, each base of every gene on the microarray has $2.5 \mathrm{x}$ or greater probe coverage. The probes $(\approx 45-60$ bases) are tiled to ensure detection of rearrangements that are as small as a few hundred bases in length. In addition, probes were selected to overlap flanking regions of repetitive sequence (e.g. guanine cytosine (GC)-rich regions). A stringent selection process was used to create an optimal probe set. The 
probes designed in silico were tested empirically against known negative and positive samples to produce a microarray CGH assay with enhanced sensitivity and specificity to detect variations in dosage.

\section{Microarray analysis}

Genomic DNA was labeled with Cy3 \& Cy5 dyes using an Enzymatic Labeling Kit (Agilent Technologies, Santa Clara, CA) according to manufacturer's instructions. Array hybridization and washing were performed on custom Agilent $8 \times 15 \mathrm{k}$ arrays (Agilent Technologies), and arrays were scanned using a High Resolution Microarray Scanner (Agilent Technologies). Analysis of the microarray data was performed in two steps. First, a proprietary software prediction program predicted the likelihood of a particular result for a given sample. Specifically, the prediction algorithm examined a window of contiguous probes, a defined subset of which must have had increased or decreased copy number to generate a positive call. Solitary probes that deviated from the baseline did not trigger a positive call by the algorithm. The software analysis was accompanied by visual review of the data.

Briefly, the average ratio (Cy5:Cy3) of fluorescent intensity obtained from cohybridized test and reference DNA for each probe was calculated and normalized. In addition to the normalizations carried out by Agilent's Feature Extraction, each probe is also normalized based on GC-content. This normalization is not necessary if all probes designed fall within tight GC-content criteria but this would not allow for tiling coverage across many genes. This normalization works by compensating for dosage biases introduced by dye normalization in probes based on their GC-content and signal intensity difference of the $\mathrm{Cy} 3$ and $\mathrm{Cy} 5$ labeled probes. The average ratios of the fluorescent intensities for each case were converted to a $\log _{2}$ scale and plotted using our proprietary software. This software normalizes each probe based on the historic performance of that probe to minimize biases attributable to the test rather than the patient sample. The theoretical $\log _{2}$ conversions for ratios (test/reference) of $1 / 2,2 / 2$, and $3 / 2$ are approximately -1 , 0 , and 0.58 respectively. In practice, the actual values never reach their theoretical limits. For single-copy losses $(1 / 2)$ and single-copy gains (3/2), we used thresholds of approximately -0.5 and 0.29 , respectively; deletions are typically visualized as probe clusters that center within the -0.5 to -1.0 amplitude lines. A cluster of probes centered between the 0.29 to 0.58 amplitude lines represents a duplication.

\section{Microarray validation study}

To validate the microarray we performed microarray analysis on samples that were previously identified to be positive or negative for a LR in BRCA1, BRCA2, MLH1,
MSH2, MSH6, EPCAM, APC, and MUTYH by multiplex quantitative PCR, MLPA, Southern blotting or longrange PCR. An additional synthetic control using genomic DNA digested with restriction enzymes was also used to assess MUTYH.

\section{Clinical testing for LRs by microarray analysis}

To examine the proportion of LRs among genetic mutations identified by a large clinical laboratory, we performed microarray analysis on patients referred to Myriad Genetic Laboratories, Inc., for clinical testing of BRCA1 and BRCA2 (May 2012 to November 2013) or $M L H 1, M S H 2, M S H 6, E P C A M$, and/or APC (September 2012 to November 2013). All patient data regarding clinical history and ancestry were obtained by health care provider report on test requisition forms. For each patient population, data were analyzed for patients who met clinical criteria predicting a relatively high probability of carrying a mutation in BRCA1 or BRCA2 (HBOC), MLH1, MSH2, MSH6 or EPCAM (Lynch syndrome) or $A P C$ (polyposis). These patients received LR analysis concurrent with sequencing analysis. Patients were excluded if microarray and sequencing analysis were not performed concurrently.

\section{Results}

\section{Validation study}

We initially validated the microarray assay for hereditary colorectal cancer genes with a blinded analysis of 357 previously tested DNA samples derived from patients with a personal or family history of CRC, of which 264 were extracted from blood and 93 from buccal samples. We correctly identified all 88 positives among 357 samples that were previously examined for deletions and duplications in $M L H 1, M S H 2, M S H 6$, and the 3' terminal region of EPCAM by multiplex quantitative PCR and/or MLPA. We also correctly identified all 7 positive samples among 307 that were previously tested for the presence of LRs in $A P C$ by Southern blot and MLPA. We correctly identified a homozygous MUTYH deletion previously detected by sequencing and confirmed by long-range PCR, as well as a synthetic positive control sample tested in replicates.

We next validated the microarray by blinded analysis of DNA samples previously examined for LR mutations in $B R C A 1$ and BRCA2 using our clinically validated multiplex quantitative PCR assay. The microarray validation was carried out in two phases and tested a total of 633 DNA samples. The second phase of validation incorporated minor enhancements for process efficiency that did not affect test sensitivity or specificity. The DNA samples examined for LRs were extracted from 317 peripheral blood and 316 buccal mouthwash specimens. Forty-two of the blood samples and 38 of the buccal samples were positive for a LR. Results for all 633 DNA samples were 100\% 
concordant with the original multiplex quantitative PCR result. In addition, these validation studies correctly identified two instances of an Alu insertion in BRCA2 specific to the Portuguese population (c.156_157insAlu). The microarray assay for BRCA1, BRCA2, MLH1, MSH2, MSH6, EPCAM, APC, and MUTYH was validated against a collective total of 990 DNA samples that were previously characterized for LRs with $100 \%$ concordance.

\section{Performance of microarray in the clinical setting}

Once validated, we performed microarray analysis on samples from patients referred for $\operatorname{HBOC}(13,124)$, Lynch syndrome $(18,498)$, and polyposis syndrome $(2,739)$ testing. All samples received LR analysis by microarray concurrent with sequencing analysis. Among all Lynch syndrome deleterious and suspected deleterious mutations detected, $17.2 \%(189 / 1,098)$ were LRs in $M L H 1, M S H 2$, MSH6, or EPCAM compared with $82.8 \%(909 / 1,098)$ sequencing mutations in $M L H 1, M S H 2$, and MSH6 (Table 1). Deletions in the 3' terminal region of EPCAM that affect expression of the adjacent $M S H 2$ gene (but not EPCAM point mutations) are associated with Lynch syndrome [22,23]. Of the LRs detected, 27.0\% (51/189) were in MLH1, $57.7 \%(109 / 189)$ in $M S H 2,5.3 \%$ (10/189) in MSH6, and $4.2 \%(8 / 189)$ in the 3 ' terminal region of EPCAM alone. Deletions in both $M S H 2$ and the 3'terminal region of EPCAM comprised 5.8\% (11/189) of the LRs. Collectively, 92.1\% (174/189) of the LRs were deletions and $7.9 \%(15 / 189)$ were duplications within $M L H 1$, MSH2, MSH6, and EPCAM.

Among all $A P C$ deleterious and suspected deleterious mutations detected, 9.6\% (31/324) were LRs while 90.4\% (293/324) were sequencing mutations. Of the LRs detected in $A P C, 93.5 \%(29 / 31)$ were deletions and $6.5 \%$ $(2 / 31)$ were duplications.

Of suspected HBOC samples tested, 1.4\% (185/13,124) had a LR mutation in BRCA1 or BRCA2. Of all HBOC deleterious or suspected deleterious mutations in $B R C A 1$ or BRCA2, $8.5 \%(185 / 2186)$ of mutations were LRs and
91.5\% (2001/2186) were sequencing mutations. Collectively, $76.8 \%(142 / 185)$ of mutations were deletions and $23.2 \%$ $(43 / 185)$ were duplications/triplications within BRCA1and BRCA2.

\section{Discussion}

We performed targeted microarray analysis concurrent with Sanger sequencing analysis on a large clinical cohort of samples with a high likelihood of HBOC or hereditary CRC mutations and showed that $17.2 \%$ of mutations in Lynch syndrome samples, $9.6 \%$ of mutations in polyposis samples, and $8.5 \%$ of mutations in HBOC samples were LRs. These results demonstrate that LRs constitute a substantial proportion of genetic mutations associated with a common hereditary cancer syndrome identified by a large clinical laboratory.

The primary advantage of targeted CGH-based microarray analysis is the ability to simultaneously detect exonlevel dosage alterations of multiple genes represented on the array. In addition, microarrays have several advantages over other commonly used molecular techniques such as Southern blotting and MLPA. Southern blotting has several limitations: it is time-consuming, expensive and requires a large amount of DNA. MLPA is a relatively inexpensive, simple and reproducible PCR-based method that uses the same equipment used for DNA sequencing $[4,24]$. MLPA is commonly used for the detection of genomic rearrangements in genes such as BRCA1 and BRCA2 [25,26]. However, MLPA has several limitations. Sequence variants in probe binding sites can yield falsepositive results; conversely, limited probe coverage across genes may produce false-negative results. Technical artifacts often necessitate confirmatory studies, further increasing cost, resources, and turnaround time. Compared to high-density microarrays, MLPA provides limited information regarding the location of the deletion or duplication breakpoints in the affected flanking/intronic regions, which may lead to laborious mapping for sequence characterization of the rearrangements. Most

Table 1 Clinical testing summary

\begin{tabular}{|c|c|c|c|c|c|}
\hline Syndrome & Gene(s) tested & Patients tested $^{\mathrm{a}}$ & $\begin{array}{l}\text { Total positive sequence } \\
\text { and LR mutations }\end{array}$ & $\begin{array}{l}\text { Total LR } \\
\text { (\% of positive mutations) }\end{array}$ & $\begin{array}{l}\text { LR by gene } \\
\text { (\% of LR) }\end{array}$ \\
\hline \multirow[t]{2}{*}{$\overline{\mathrm{HBOC}}$} & $B R C A 1$ & 13,124 & 2,186 & $185(8.5 \%)$ & $167(90.3 \%)$ \\
\hline & $B R C A 2$ & & & & $18(9.7 \%)$ \\
\hline \multirow[t]{5}{*}{ Lynch syndrome } & MLH1 & 18,498 & 1,098 & 189 (17.2\%) & $51(27.0 \%)$ \\
\hline & MSH2 & & & & 109 (57.7\%) \\
\hline & MSH6 & & & & 10 (5.3\%) \\
\hline & EPCAM & & & & $8(4.2 \%)$ \\
\hline & EPCAM/MSH2 & & & & $11(5.8 \%)$ \\
\hline Polyposis & $A P C$ & 2,739 & 324 & $31(9.6 \%)$ & 31 (100\%) \\
\hline
\end{tabular}

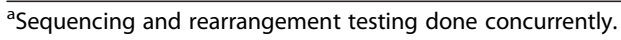


MLPA reagents are available as kits designed to analyze one or a few genes at a time. The requirement for manual processing further increases sample turnaround time and prohibits high-throughput applications, which are crucial for high-volume sample processing in large clinical laboratories. MLPA also has the potential to miss some partial deletions or duplications. Figure 1 illustrates the extent of probe coverage for $\mathrm{MSH} 2$ exon 10 in our targeted microarray assay. The MLPA assay did not detect this deletion because the single MLPA probe hybridizes to a region of MSH2 intron 10, which is not deleted. A typical MLPA assay will have 1-2 probes per exon, depending on the size of that exon. Thus, any dosage changes that alter a part of the exon not covered by the MLPA probe will not be detected. Furthermore, it is notable that our targeted microarray probe coverage extends into the binding sites for our sequencing primers, as depicted in Figure 1. In this way, we are able to detect deletions that remove sequencing primer binding sites and lead to mono-allelic sequencing results.

Figure 2 shows the actual partial deletion of exon 10 in $\mathrm{MSH} 2$ in one of our patients, detected by microarray analysis. The microarray showed a loss of probes spanning part of the exon. Confirmatory long-range PCR and sequencing analysis demonstrated that the deletion removed approximately 2,000 base pairs (bp) of sequence (data not shown).

Microarray-CGH is less prone to technical artifacts and is amenable to automation, which allows microarray to be utilized as a high-throughput assay that can decrease sample turnaround times and costs. Microarrays are also scalable-probes can easily be added to current areas of interest and additional syndromes/genes can be added at little additional cost to the laboratory. Targeted microarray CGH therefore provides yet another advantage over other LR detection methodologies. Microarray can be easily adapted for use as an adjunct for panel analysis by NGS, allowing the laboratory to evaluate the LR status of multiple genes at once.

Because microarrays are designed to detect gains and losses in dosage at a specific locus, insertions of material from elsewhere in the genome, particularly of repetitive sequence elements, are difficult to detect. However, our results suggest insertions occur infrequently. To date, the most common of all known insertions found in BRCA1 and $B R C A 2$ is an Alu insertion that integrates within nucleotides 156 and 157 in exon 3 of the BRCA2 gene (c.156_157insAlu). This has been reported as a founder mutation in the Portuguese population. We have specifically designed probes on our microarray assay to screen for the Portuguese founder mutation. We have included probes specific to the native $B R C A 2$ region in exon 3 that would be disrupted by the insertion as well as other probes that hybridize to the Alu inserted sequence. For this latter probe set, one half of the probe hybridizes to the native $B R C A 2$ exon 3 sequence and the other half hybridizes to the first 30 bases of the inserted sequence. Together, this collection of probes functions as a screening tool to flag samples that potentially carry the Portuguese insertion. We confirm the presence of the insertion using a targeted breakpoint-specific PCR assay. During the study period, we identified only three cases of the BRCA2 (c.156_157insAlu) founder mutation in 13,124 clinical samples tested.

Finally, microarrays are not reliant on clinical suspicion of a specific condition. They provide an objective, comprehensive means of genetic diagnosis. In particular, Lynch syndrome can be the most challenging hereditary CRC syndrome to recognize because of the absence of an overt polyposis phenotype, and there are many patients with Lynch syndrome who remain undiagnosed. Testing strategies such as microarray analysis that

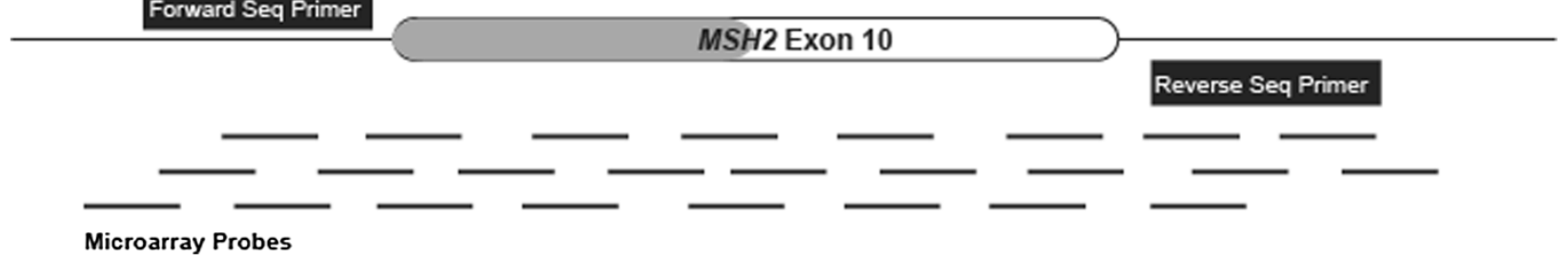

Figure 1 Diagrammatic representation of the 1900 bp deletion that starts in intron 9 of MSH2 and removes the first 94 bases of exon 10. This figure maps the locations of multiple overlapping microarray probes that can assess for LRs in this region. The MLH1/MSH2 MLPA kit (MRC-Holland, P003-C1) has only one MLPA probe in intron 10. The locations of the forward and reverse sequencing primers and the microarray probes that cover these regions are also depicted. 


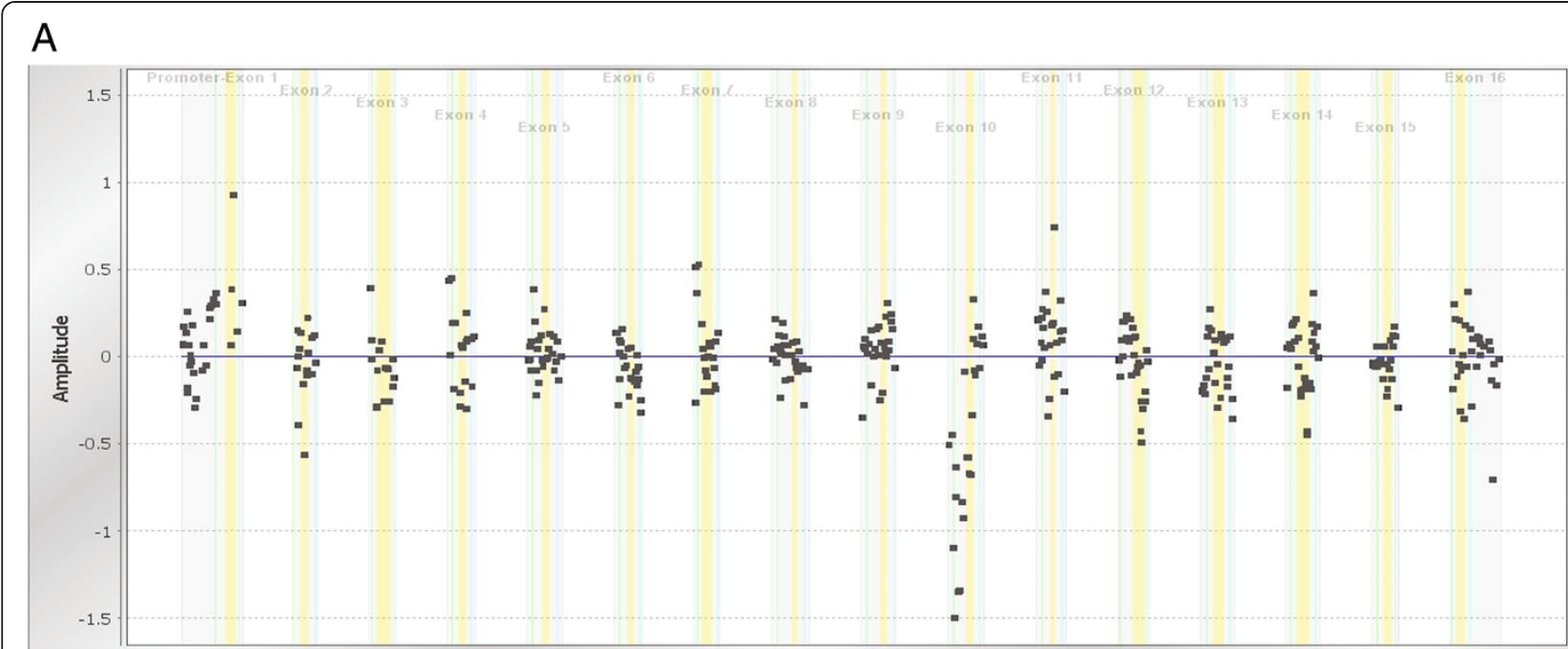

B

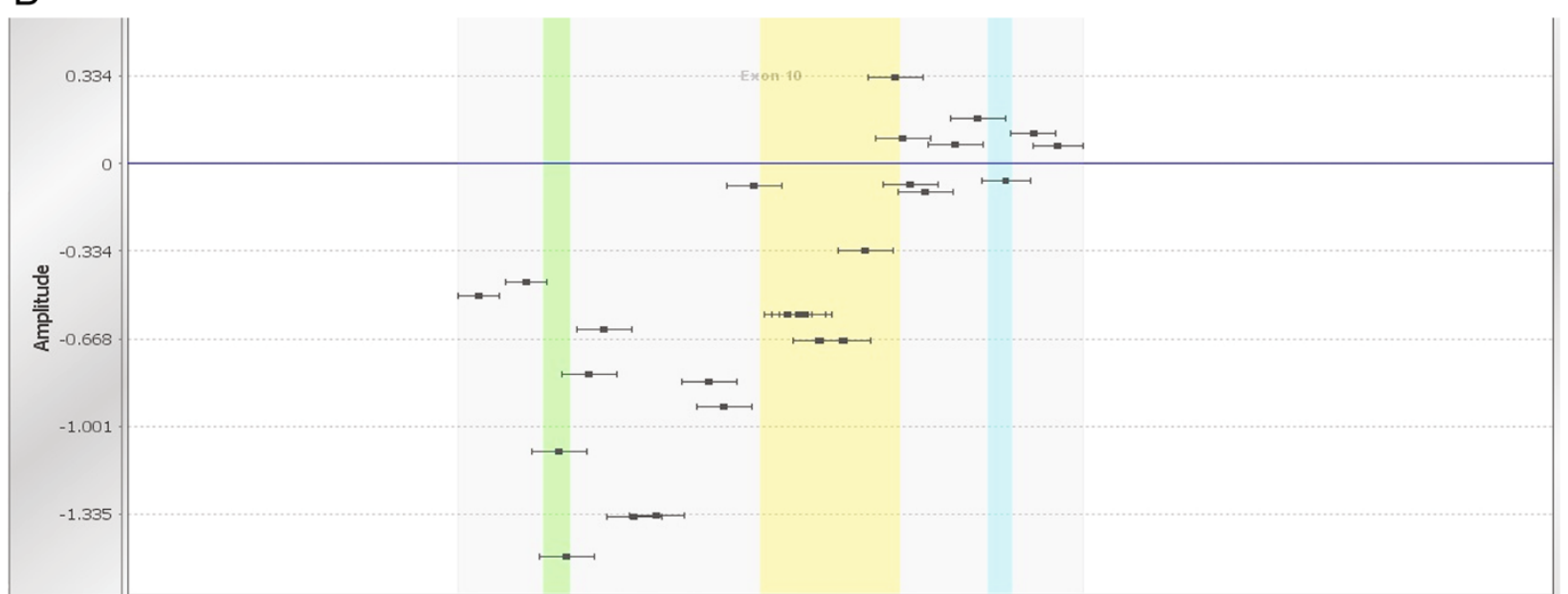

Figure 2 Targeted microarray result demonstrating a partial deletion of exon 10 in $M S H 2$ at the A) gene level and B) probe level.

Vertical yellow lines represent MSH2 exons. Individual probes are represented by the black dots. Probe clusters for exons devoid of LRs are shown to center at the 0 horizontal line, which represents two allelic copies. The cluster of microarray probes spanning the $5^{\prime}$ portion of MSH2 exon 10 probe centers at approximately -0.75 , indicating the presence of a partial deletion of this exon.

interrogate LRs will help identify additional patients with a hereditary cancer syndrome, particularly those for whom sequencing analysis was negative.

The ability to analyze multiple genes simultaneously facilitates this shift away from single-locus genetic testing toward panel testing of multiple genes of interest. With the advent of NGS, our laboratory and others are working towards adapting this technology for the detection of LRs as well.

\section{Conclusion}

At present, we have validated the clinical use of microarray CGH to augment LR analysis in our hereditary cancer panel of 25 genes associated with multiple adult-onset hereditary cancers. Once NGS is established as a standard method for the accurate detection of LRs, microarrays may retain utility for confirmatory testing. Panel-based testing strategies will help identify additional patients with a hereditary cancer syndrome and enable clinicians to better manage individuals and families who are affected or at risk for these inherited disorders with specific genetic and clinical counseling, screening, and treatment recommendations.

\section{Abbreviations}

LR: Large rearrangement; HBOC: Hereditary breast and ovarian cancer; CGH: Comparative genomic hybridization; CRC: Colorectal cancer; MMR: Mismatch repair; FAP: Familial adenomatous polyposis;

PCR: Polymerase chain reaction; MLPA: Multiplex ligation-dependent probe 
amplification; NGS: Next-generation sequencing; GC: Guanine cytosine; bp: Base pairs.

\section{Competing interests}

All authors are employees of Myriad Genetic Laboratories, Inc. and receive salary and stock options as compensation for employment.

\section{Authors' contributions}

DM-D, TJ, NW, CB, JS, MR, AM, AT, JH, JC, CA, KM, and BR contributed to the design of the study. DM-D, KM, CA, NG, TJ, and BR carried out the interpretation of the data. DM-D, BR, TJ, and AT participated in the creation of the manuscript. All authors read and provided final approval for the version to be published.

\section{Acknowledgements}

We would like to thank Sonia Chen, Marie Balzotti, Carrie Colvin, Jeffrey Trost, Lynn Anne Burbidge, and Eric Rosenthal for their contribution to and input on the data in this manuscript, the laboratory personnel at Myriad Genetic Laboratories, Inc. for their work, and Kirstin Roundy for her assistance with figure preparation, manuscript editing and submission. We would also like to acknowledge and thank the healthcare providers and patients who have participated in Myriad Genetic Laboratories' Variant Classification Program.

Received: 22 May 2014 Accepted: 29 August 2014

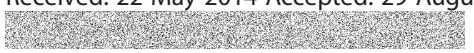

\section{References}

1. Kastrinos F, Stoffel EM: History, genetics, and strategies for cancer prevention in Lynch syndrome. Clin Gastroenterol Hepatol 2014, 12:715-727. quiz e741-713.

2. Li D, Hu F, Wang F, Cui B, Dong X, Zhang W, Lin C, Li X, Wang D, Zhao Y: Prevalence of pathological germline mutations of hMLH1 and hMSH2 genes in colorectal cancer. PLoS One 2013, 8:e51240.

3. Charbonnier F, Olschwang S, Wang Q, Boisson C, Martin C, Buisine MP, Puisieux A, Frebourg T: MSH2 in contrast to MLH1 and MSH6 is frequently inactivated by exonic and promoter rearrangements in hereditary nonpolyposis colorectal cancer. Cancer Res 2002, 62:848-853.

4. Gille JJ, Hogervorst FB, Pals G, Wijnen JT, van Schooten RJ, Dommering CJ, Meijer GA, Craanen ME, Nederlof PM, de Jong D, McElgunn CJ, Schouten JP, Menko FH: Genomic deletions of MSH2 and MLH1 in colorectal cancer families detected by a novel mutation detection approach. $\mathrm{Br} J$ Cancer 2002, 87:892-897.

5. Taylor CF, Charlton RS, Burn J, Sheridan E, Taylor GR: Genomic deletions in $\mathrm{MSH} 2$ or MLH1 are a frequent cause of hereditary non-polyposis colorectal cancer: identification of novel and recurrent deletions by MLPA. Hum Mutat 2003, 22:428-433.

6. Wagner A, Barrows A, Wijnen JT, van der Klift H, Franken PF, Verkuijlen P, Nakagawa H, Geugien M, Jaghmohan-Changur S, Breukel C, Breukel C, Meijers-Heijboer H, Morreau H, van Puijenbroek M, Burn J, Coronel S, Kinarski Y, Okimoto R, Watson P, Lynch JF, de la Chapelle A, Lynch HT, Fodde R: Molecular analysis of hereditary nonpolyposis colorectal cancer in the United States: high mutation detection rate among clinically selected families and characterization of an American founder genomic deletion of the MSH2 gene. Am J Hum Genet 2003, 72:1088-1100.

7. Wang Y, Friedl W, Lamberti C, Jungck M, Mathiak M, Pagenstecher C, Propping $P$, Mangold E: Hereditary nonpolyposis colorectal cancer: frequent occurrence of large genomic deletions in MSH2 and MLH1 genes. Int J Cancer 2003, 103:636-641.

8. Grabowski M, Mueller-Koch Y, Grasbon-Frodl E, Koehler U, Keller G, Vogelsang H, Dietmaier W, Kopp R, Siebers U, Schmitt W, Neitzel B, Gruber M, Doerner C, Kerker B, Ruemmele P, Henke G, Holinski-Feder E: Deletions account for 17\% of pathogenic germline alterations in $\mathrm{MLH} 1$ and $\mathrm{MSH} 2$ in hereditary nonpolyposis colorectal cancer (HNPCC) families. Genet Test 2005, 9:138-146.

9. Pino MS, Chung DC: Application of molecular diagnostics for the detection of Lynch syndrome. Expert Rev Mol Diagn 2010, 10:651-665.

10. Baudhuin LM, Ferber MJ, Winters JL, Steenblock KJ, Swanson RL, French AJ, Butz ML, Thibodeau SN: Characterization of hMLH1 and hMSH2 gene dosage alterations in Lynch syndrome patients. Gastroenterology 2005, 129:846-854.

11. Puget N, Torchard D, Serova-Sinilnikova OM, Lynch HT, Feunteun J, Lenoir GM, Mazoyer S: A 1-kb Alu-mediated germ-line deletion removing BRCA1 exon 17. Cancer Res 1997, 57:828-831.
12. Palma MD, Domchek SM, Stopfer J, Erlichman J, Siegfried JD, TiggesCardwell J, Mason BA, Rebbeck TR, Nathanson KL: The relative contribution of point mutations and genomic rearrangements in BRCA1 and BRCA2 in high-risk breast cancer families. Cancer Res 2008, 68:7006-7014.

13. Judkins T, Rosenthal E, Arnell C, Burbidge LA, Geary W, Barrus T, Schoenberger J, Trost J, Wenstrup RJ, Roa BB: Clinical significance of large rearrangements in BRCA1 and BRCA2. Cancer 2012, 118:5210-5216.

14. Pinkel D, Segraves R, Sudar D, Clark S, Poole I, Kowbel D, Collins C, Kuo WL, Chen C, Zhai Y, Dairkee SH, Ljung BM, Gray JW, Albertson DG: High resolution analysis of DNA copy number variation using comparative genomic hybridization to microarrays. Nat Genet 1998, 20:207-211.

15. Redon R, Carter NP: Comparative genomic hybridization: microarray design and data interpretation. Methods Mol Biol 2009, 529:37-49.

16. Vissers LE, Veltman JA, van Kessel AG, Brunner HG: Identification of disease genes by whole genome CGH arrays. Hum Mol Genet 2005, Spec No. 2:R215-R223.

17. Albertson DG, Pinkel D: Genomic microarrays in human genetic disease and cancer. Hum Mol Genet 2003, 12 Spec No 2:R145-R152.

18. Kanamori M, Sano A, Yasuda T, Hori T, Suzuki K: Array-based comparative genomic hybridization for genomic-wide screening of DNA copy number alterations in aggressive bone tumors. J Exp Clin Cancer Res 2012, 31:100.

19. Staaf J, Torngren T, Rambech E, Johansson U, Persson C, Sellberg G, Tellhed $L$, Nilbert $M$, Borg $A$ : Detection and precise mapping of germline rearrangements in BRCA1, BRCA2, MSH2, and MLH1 using zoom-in array comparative genomic hybridization (aCGH). Hum Mutat 2008, 29:555-564.

20. Rouleau E, Lefol C, Tozlu S, Andrieu C, Guy C, Copigny F, Nogues C, Bieche I, Lidereau R: High-resolution oligonucleotide array-CGH applied to the detection and characterization of large rearrangements in the hereditary breast cancer gene BRCA1. Clin Genet 2007, 72:199-207.

21. Alkan C, Coe BP, Eichler EE: Genome structural variation discovery and genotyping. Nat Rev Genet 2011, 12:363-376.

22. Ligtenberg MJ, Kuiper RP, Chan TL, Goossens M, Hebeda KM, Voorendt M, Lee TY, Bodmer D, Hoenselaar E, Hendriks-Cornelissen SJ, Tsui WY, Kong CK, Brunner HG, van Kessel AG, Yuen ST, van Krieken JH, Leung SY, Hoogerbrugge $\mathrm{N}$ : Heritable somatic methylation and inactivation of $\mathrm{MSH} 2$ in families with Lynch syndrome due to deletion of the 3' exons of TACSTD1. Nat Genet 2009, 41:112-117.

23. Kovacs ME, Papp J, Szentirmay Z, Otto S, Olah E: Deletions removing the last exon of TACSTD1 constitute a distinct class of mutations predisposing to Lynch syndrome. Hum Mutat 2009, 30:197-203.

24. Schouten JP, McElgunn CJ, Waaijer R, Zwijnenburg D, Diepvens F, Pals G: Relative quantification of 40 nucleic acid sequences by multiplex ligation-dependent probe amplification. Nucleic Acids Res 2002, 30:e57.

25. Vasickova P, Machackova E, Lukesova M, Damborsky J, Horky O, Pavlu H, Kuklova J, Kosinova V, Navratilova M, Foretova L: High occurrence of BRCA1 intragenic rearrangements in hereditary breast and ovarian cancer syndrome in the Czech Republic. BMC Med Genet 2007, 8:32.

26. Rodriguez M, Torres A, Borras J, Salvat M, Guma J: Large genomic rearrangements in mutation-negative BRCA families: a population-based study. Clin Genet 2010, 78:405-407.

\section{doi:10.1186/s13046-014-0074-9}

Cite this article as: Mancini-DiNardo et al: Design and validation of an oligonucleotide microarray for the detection of genomic rearrangements associated with common hereditary cancer syndromes. Journal of Experimental \& Clinical Cancer Research 2014 33:74. 